\title{
DESDynI Lidar for Solid Earth Applications
}

\author{
Jeanne Sauber ${ }^{1}$, Michelle Hofton ${ }^{2}$, Ronald Bruhn ${ }^{3}$, Scott Luthcke' ${ }^{1}$, and Bryan Blair \\ ${ }^{1}$ NASA Goddard Space Flight Center, Greenbelt, MD 20771 \\ ${ }^{2}$ University of Maryland, College Park, MD 20742 \\ ${ }^{3}$ University of Utah, Salt Lake City, UT 84112
}

\begin{abstract}
As part of the NASA's DESDynI mission, global elevation profiles from contiguous $25 \mathrm{~m}$ footprint Lidar measurements will be made. Here we present results of a performance simulation of a single pass of the multi-beam Lidar instrument over uplifted marine terraces in southern Alaska. The significance of the Lidar simulations is that surface topography would be captured at sufficient resolution for mapping uplifted terraces features but it will be hard to discern 1-2m topographic change over features less than tens of meters in width. Since Lidar would penetrate most vegetation, the accurate bald Earth elevation profiles will give new elevation information beyond the standard 30-m DEM.
\end{abstract}

\section{INTRODUCTION}

Although the primary objective of the DESDynl-Lidar is the recovery of vegetation structure for estimation of above ground biomass and for ecosystem health, the $25 \mathrm{~m}$ footprint, contiguous multi-beam data [1] will provide important new information on surface topography and topographic change. Over the last decade we have conducted a number of pre- and post-launch studies that support the rationale for the Lidar simulations reported here. For example, prior to the launch of the Ice, Cloud and land Elevation laser altimeter satellite (ICESat, single laser beam with $55-80 \mathrm{~m}$ footprints), we used NASA's Airborne Topographic Mapper [2] (ATM) to estimate how effective individual ICESat footprint data from exact repeat satellite tracks would be in recovering ice elevation change over the rapidly retreating Jakobshavn Glacier. These simulations enabled us to identify an approach to successfully use ICESat plus InSAR derived digital elevation models (DEM) for ice elevation change on mountain glaciers [3,4] Also, we have used repeat Laser Vegetation Imaging Sensor (LVIS) measurements with full-waveform, 20-m contiguous footprints, to quantify pyroclastic and lava flow of a stratovolcano in Costa Rica[5]. In another study, near Barrow, Alaska, we introduced high quality ICESat derived positions into processing of ERS-1/2 data to improve the vertical resolution of the InSAR derived DEM by a factor of three [6]. An extension of this approach to fuse the moderate resolution multi-beam DESDynI-Lidar into processing of SAR data for derivation of DEM for a wider range of topographic slope and roughness could be especially useful outside SRTM coverage.

The complex plate boundary in southern coastal Alaska provides an excellent setting for testing DESDynI capabilities to recover fundamental parameters of seismotectonic processes. Also, aircraft acquired Lidar and SAR data have been acquired in this region in the last decade that can be used for simulations. Here we use the National Center for Airborne Laser Mapping lidar (NCALM) data acquired over marine terraces west of Icy Bay ("Sullivan data") in southern Alaska in September 2005 as part of the NSF STEEP study (www.ig.utexas.edu/steep). In this paper, we report on the simulation studies of the multi-beam Lidar instrument performance over uplifted marine terraces, active faults and folds, and possible uplift associated with the 1899 Yakataga seismic event $(\mathbf{M}=8)$ in southern, coastal Alaska.

\section{APPROACH}

In this performance study we created synthetic waveforms by extracting the NCALM point cloud data along one set of the DESDynI-Lidar calculated ground tracks (Fig. 1). We used a single pass of DESDynI-Lidar satellite ground tracks with 5 laser beams and across track spacing of $\sim 850 \mathrm{~m}$ to geolocate the simulated waveforms. Each ground track had individual laser returns spaced $30 \mathrm{~m}$ along track (Fig. 1).

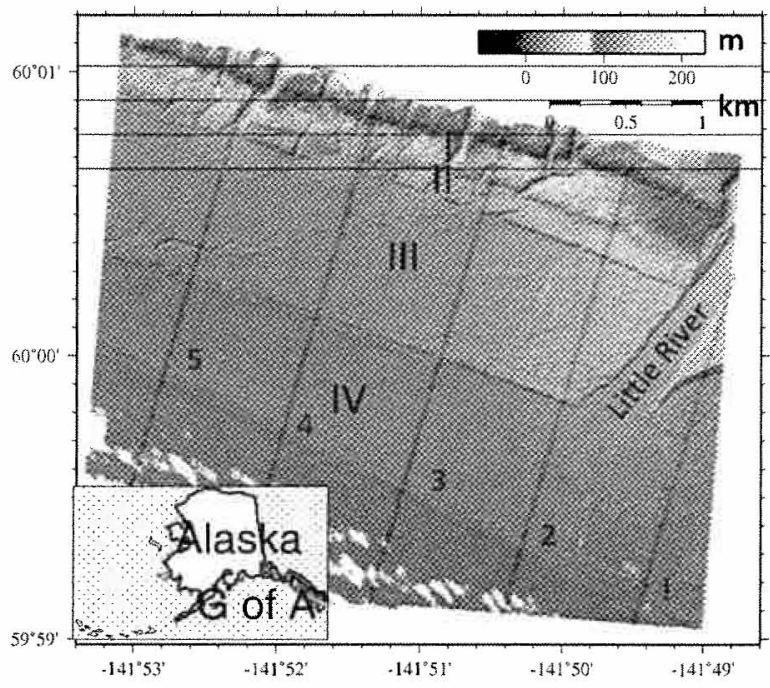

Fig. 1. NCALM-derived topographic map of study region showing the marine terrace surfaces (I-IV) and the DESDynl-Lidar (NE-SW) ground tracks. The offset between terrace surface $\mathrm{II}$ and $\mathrm{III}$ is frequently masked by alluvial deposits. A subtle offset of 1-2 $\mathrm{m}$ between terrace IV and III may be due to coseismic displacement associated with the $1899 \quad \mathbf{M}=8$ earthquake. The northern portion of profile \#1 crosses the Little River and is not included. Inset: outline of Alaska with study area. 
By accounting for sensor characteristics such as footprint size and the temporal and spatial distribution of laser energy, we generated pseudo-DESDynI waveforms from the last return point cloud data (Fig. 2) using the approach developed earlier for aircraft LVIS data [7]. Elevation measurements are extracted using information obtained in the waveform only. The lowest and highest elevations within each footprint correspond to the lowest and highest detected signals above a predefined threshold. The centroid of the waveform has also been extracted to investigate the precision and accuracy of this as an elevation measurement over the terrace surfaces. These waveform derived metrics have been compared to corresponding small-footprint elevations to assess their precision and accuracy.

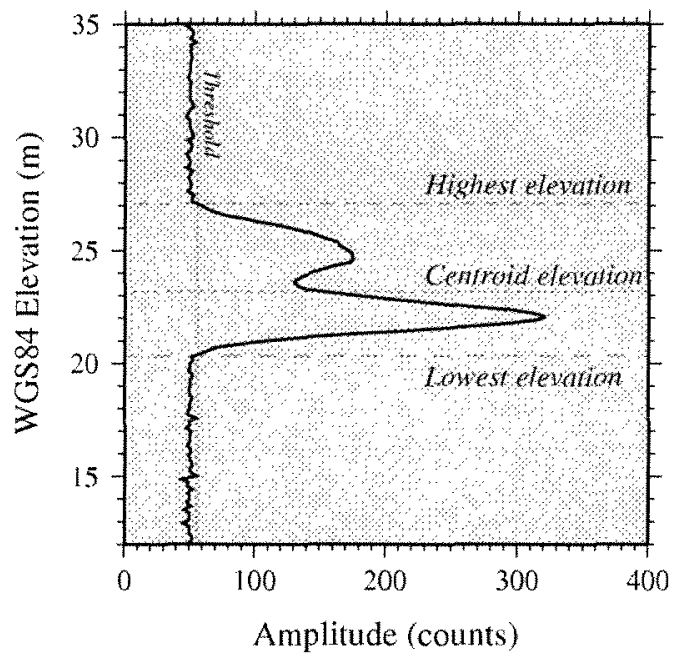

Fig. 2. WGS84 elevation versus waveform amplitude for a simulated waveform over a footprint located along Track 1 near the transition from near-coastal terrace level IV to the next terrace level. The waveform has been simulated assuming DESDynI-like parameters for the observations (i.e., laser pulse width (9ns), laser footprint diameter $(25 \mathrm{~m})$, waveform vertical resolution ( $2 \mathrm{~ns})$ ). Random noise ( 2 counts) was also included to simulate background noise present in the measurement. The range between the lowest and highest elevation could be due to a variable ground surface or NCALM Lidar returns from low-lying brush (higher curve).

\section{GEODETIC IMAGING OF SEISMOTECTONIC STRUCTURES IN SOUTHERN ALASKA}

Between Icy Bay and Cape Yakataga there are four marine terraces that range in elevation from approximately 1 $\mathrm{m}$ to $50 \mathrm{~m}$ located on the southern flank of the Sullivan anticline, and in the footwall of the Sullivan thrust fault ${ }^{8}$. These features trend roughly east-west, a nearly optimum orientation for future roughly N-S DESDynI-Lidar ground tracks (Fig. 1). The terraces contain evidence for rapid uplift during large to great magnitude earthquakes [8]. Terrace surfaces are hundreds of meters to over a kilometer in width, partly buried by alluvial fans, and separated by remnant sea cliffs that mark the former shorelines. The
Sullivan fault is marked by a prominent escarpment on the mountain above the highest terrace level; this feature is more evident to the west of the region shown in Fig. 1. The thick coastal forest and brush mask important aspects of the geomorphology on aerial photographs, standard DEM, and C-band synthetic aperture radar (SAR) images, a problem that is largely overcome by the NCALM Lidar data [9]: An advantage of even the medium footprint DESDynI-Lidar data over the standard existing $30 \mathrm{~m} \mathrm{DEM}$ is the ability to see through the vegetation to discern the individual terrace heights, features of the escarpment associated with the Sullivan fault, and to map out the morphology of the Sullivan anticline. Additionally, the DESDynI-Lidar derived elevation profiles will be provided in a common global reference frame. This will facilitate fusion of this data with other DEM and satellite images.

\section{A. Centroid elevations along five DESDynl-Lidar ground tracks}

The centroid elevation for each footprint has been used to create profiles along the 5 tracks shown in Fig. 1. For example, the Track 2 and 3 profiles (from southwest to northeast) in Fig. 3 show offset (in red) of the distinct sloped surfaces (blue lines) associated with the 4 different terraces.
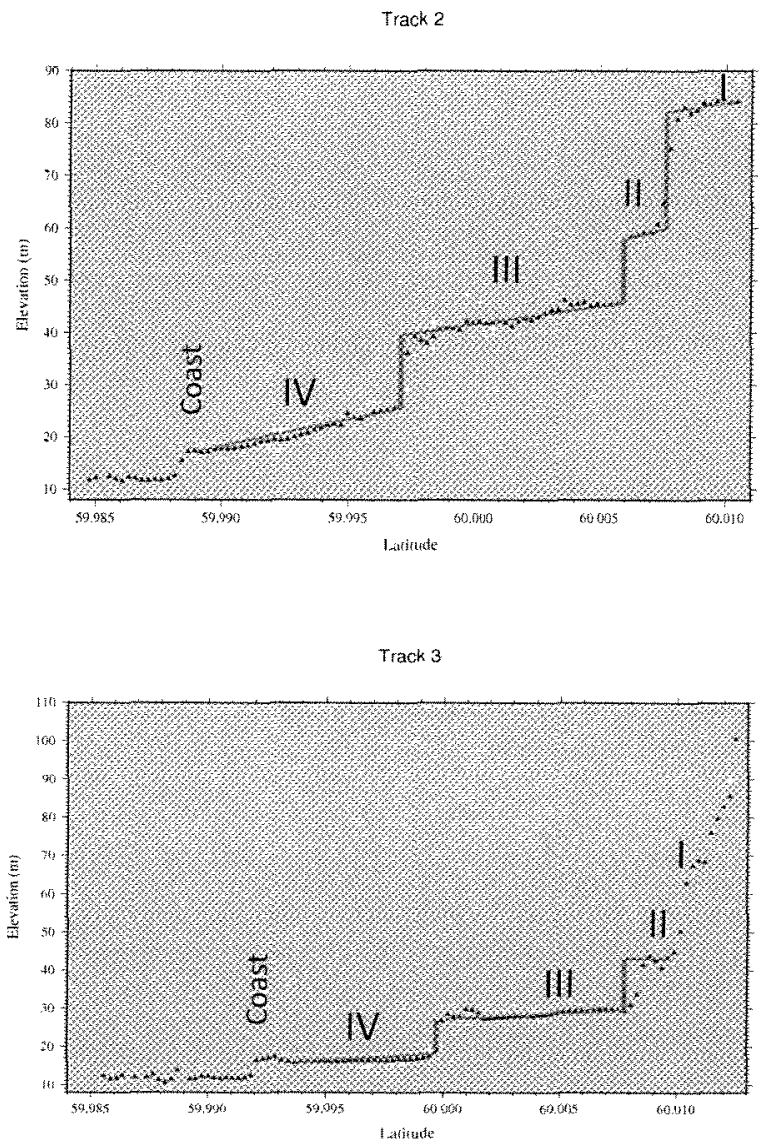

Fig. 3 Elevation profiles along Track 2 (top) and Track 3 (bottom) as a function of latitude with estimated offset (red) and terrace surface slope (blue). 
We examined the centroid elevation plotted in Fig. 3 (blue diamond) compared to the highest and lowest elevation (above the background noise threshold) from the NCALM data. The elevation differences over the flat terrace surfaces were generally $2-4 \mathrm{~m}$ but over rough areas this difference could be larger (up to $9+\mathrm{m}$ ). For the fairly flat terrace surfaces, the centroid elevation was generally in the middle of the upper and lower elevation bounds from the NCALM data. As expect for a $25 \mathrm{~m}$ footprint, the upper and lower bounds transition regions over faults or the terraces were large and variable relative to the centroid.

The large offsets between individual terrace levels as well as the slope trends across terraces in a common reference frame enable more robust comparisons. For example, the terrace offsets are largest in Track 2 and then the offsets become more muted to the west, which cross alluvial fans that have formed on the lower terrace level following uplift, and partly bury the original sea cliffs (Fig. 1 and 3). In general, the individual terraces occur at lower elevations on the more westerly tracks, this partly caused by a lateral decrease in elevation away from the high points, or apices of a temporal sequence of alluvial fans that built out from Little River onto each terrace level. Only after accounting for lateral variations in alluvial fan aggradation on the terrace surfaces can we search for evidence of deformation and uplift related to earthquake slip or post-glacial uplift episodes to the east near Icy Bay.

The significance of the DESDynI-Lidar simulations is that surface topography is captured at sufficient resolution for mapping uplifted marine terraces, including the alluvial fans that built out and buried the marine deposits following uplift. Relic sea cliffs at the back of the terraces are also resolved as abrupt elevation steps with topographic relief dictated by the amount of post uplift burial by alluvial processes, and to a lesser degree erosional dissection. Although the moderate-scale topographic features are captured, uplift on features such as the 1899 berm that are just several tens of meters across in width are hard to discern in the DESDynI-Lidar elevation profiles.

\section{B. Future steps in waveform simulation over the terraces}

To simulate the DESDynI-Lidar waveforms from aircraft point cloud Lidar data, returns from all surfaces should be included as the full waveform representation is an important feature for identifying vegetation structure. The NCALM data retains the first and last Lidar returns. In the next step of our simulation we will include both the first and last returns. We will estimate the ground elevation beneath any canopy using an automatic search algorithm. As mentioned in the Fig. 2 caption, the first (highest) curve may actually be reflections from low-lying brush. An advantage of full waveform Lidar systems such as LVIS and DESDynI-Lidar is that is it easier to associate the various returns with known vegetation structure.

\section{Ongoing simulations over fold and fault structures covered by glaciers}

In the late summer of $2005, A T M$ laser swath $(\sim 480 \mathrm{~m}$ width) data [2] was acquired in coastal, southeastern Alaska and it included data from the Malaspina and Seward glaciers (https://www.atm.wallops.gov). We used a version of this data (ICESSN standard nadir product) with a larger footprint size $(80 \mathrm{~m})$ to examine the ice characteristics along the ATM profile. ... In the lower Seward the glacier surface elevation, along track slope, and within footprint roughness and slope were used to identify thrust faults beneath the ice. These results are being used to interpret ice velocities derived from ALOS PALSAR L-Band InSAR data [9]

\section{COSEISMIC SLIP RECOVERY SCENARIO FOR A SOUTHERN}

\section{ALASKA EARTHQUAKE}

As summarized in the DESDynI Science Definition Document, "in areas where the surface is disrupted over the course of a large geophysical event such as coseismic displacement and shaking of the surface, the InSAR-derived surface deformations measurements cannot be made. It is helpful to take advantage of the Lidar to repeat elevation transects over the deformed surface to fill in some area of disruption." [10] As summarized above, we simulated waveforms along DESDynI-Lidar ground tracks over the uplifted region associated earlier great earthquakes with just one DESDynI-Lidar pass assuming a 5 laser beam configuration. To estimate coseismic displacements following a large earthquake, it would useful to use an existing pre-earthquake DEM along with the DESDynI Lidar over the region. Alternatively, if an off-nadir pointing capability exits, repeat targeting of the near-field coseismic deformation region would increase the number of transects over a short-coseismic interval. This ongoing study is enabling us to assess the minimum number of transects needed to provide timely, and spatially dense enough, data for estimation of earthquake parameters to calculate associated stress redistribution and triggered slip on nearby faults, inform detailed field studies, and to enable disaster managers to assess damage as discussed in the "Use Case Scenario" for a Seismic Event in California outlined in the Report of the DESDynI Applications Workshop [11].

\section{SUMMARY}

Since DESDynI-Lidar would penetrate most vegetation, the accurate bald Earth elevation profiles will give new information beyond the standard 30-m DEM. In our southern Alaska study region, the major crustal seismotectonic features are roughly perpendicular to the satellite ground tracks and the accurate elevation profiles are enabling process studies of folds, faults, and marine terraces. For a major earthquake with on-land surface disruption, such as a thrust-slip event in southern Alaska, DESDynI-Lidar could provide new and timely near-field vertical static displacements when used in combination with a pre-earthquake acquired DEM or with some off-nadir pointing to previous DESDynI-Lidar transects. We have found that these new simulations inform prelaunch 
strategies for maximizing our ability to expand the range of solid Earth applications for this medium resolution Lidar as part of the DESDynI mission.

\section{REFERENCES}

[1] J.B. Blair, J. Ranson, R. Dubayah, B Coyle, and C. Salerno, Multibeam lidar instrument design, measurement capabilities, and technical readiness, Eos Trans. $A G U, 88(52)$, Fall Meet. Suppl., Abstract G54A-03, 2007.

[2] W. B. Krabill, et al., Aircraft laser altimetry of elevation changes of the Greenland ice sheet, technique and accuracy assessment, $J$. of Geodynamics, 34, 357-376, 2002

[3] J. Sauber, B. Molnia, C. Carabajal, S. Luthcke, and R. Muskett, Ice elevations and surface change on the Malaspina Glacier, Alaska, Geophys. Res. Lett., 32, L23S01, doi:10.1029/2005GL023943, 2005.

[4] R. R. Muskett, C.S. Lingle, J.M. Sauber, A.S. Post, W.V. Tangborn, B.T. Rabus, K.A. Echelmeyer, Airborne and spaceborne DEM- and laser altimetry-derived surface elevation and volume changes of the Bering Glacier system, Alaska, USA, and Yukon, Canada, $J$, of Glaciology, 55(190), 1972-200, 2009.

[5] M. A. Hofton, E. Malavassi, and J. B. Blair, Quantifying recent pyroclastic and lava flows at Arenal Volcano, Costa Rica, using medium-footprint lidar, Geophy. Res. Lett., 33, L21306, doi:10.1029/2006GL027822, 2006.

[6] D. K. Atwood, R. M. Guritz, R. R. Muskett, C. S. Lingle, J. M. Sauber, and J. T. Freymueller, DEM Control in Arctic Alaska with ICESat Laser Altimetry, IEEE Trans. on Geoscience and Remote Sensing, 45(11), 3710-3720, 2007

[7] J.B. Blair and M.A. Hofton, Modeling large footprint laser altimeter returns using high-resolution elevation data, Geophys. Res. Lett. 26:2509-2512, 1999

[8] I. Shennan, R. Bruhn, and G. Plafker, Multi-segment earthquakes and tsunami potential of the Aleutian megathrust, OSR, 28(1), 7-13, 2009.

[9] R.L. Bruhn, J.M.Sauber, R.R. Forster, and M.M. Cotton, GeosphereCryosphere interactions in the Saint Elias orogen, Alaska and Yukon, Eos Trans. AGU, Fall Meeting Suppl. Abstract G52B-94, 2009.

[10] DESDynl Science Study Group, Deformation, Ecosystem Structure and Dynamics of Ice (DESDynl), A NASA Mission in the NRC Decadal Survey Earth Science Applications from Space, Science Definition Document, R. Dubayah, B. Hager, and I. Joughin, cochairs, 2009.

[11] A. Donnellan, G. Bawden, and J. Rundle, Report of the DESDynI Application Workshop, 2009. 